\title{
Gestational diabetes, pre-pregnancy obesity and pregnancy weight gain in relation to excess fetal growth: variations by race/ethnicity
}

\author{
K. Bowers $•$ S. K. Laughon $\cdot$ M. Kiely $・$ J. Brite $\cdot$ Z. Chen $\cdot$ C. Zhang
}

Received: 4 September 2012 / Accepted: 18 February 2013 /Published online: 10 April 2013

(C) Springer-Verlag (outside the USA) 2013

\begin{abstract}
Aims/hypothesis The escalating rate of childhood obesity is a public health concern worldwide, with children in certain ethnic groups being disproportionately affected. Our objective was to examine the joint effects of pre-pregnancy adiposity, pregnancy weight gain and gestational diabetes (GDM) in relation to excess fetal growth and to identify susceptible races or ethnic populations.

Methods The risk for delivery of a large-for-gestational-age (LGA) infant, specific to race and fetal sex, was evaluated in 105,985 pregnancies in the Consortium on Safe Labor from 2002-2008. Generalised estimating equations were used to estimate the risk for delivery of LGA infants. Joint effects were employed to evaluate the interplay of three risk factors.
\end{abstract}

K. Bowers $\cdot$ S. K. Laughon $\cdot$ M. Kiely $\cdot$ C. Zhang $(\square)$ Epidemiology Branch, Division of Epidemiology, Statistics, and Preventive Research, Eunice Kennedy Shriver National Institute of Child Health and Human Development, 6100 Executive Blvd, Rockville,

MD 20852, USA

e-mail: zhangcu@mail.nih.gov

J. Brite

CUNY Institute of Demographic Research and CUNY School of Public Health at Hunter College City University of New York, New York, NY, USA

Z. Chen

Biostatistics Branch, Division of Epidemiology, Statistics, and Preventive Research, Eunice Kennedy Shriver National Institute of Child Health and Human Development, Rockville, MD, USA

Present address:

K. Bowers $(\square)$

Division of Biostatistics and Epidemiology,

Cincinnati Children's Hospital Medical Center,

3333 Burnet Avenue, MLC 5041, Cincinnati, OH 45229, USA

e-mail: katherine.bowers@cchmc.org
Models were stratified by racial group considering one, two or three factors (i.e. pre-pregnancy adiposity, pregnancy weight gain and GDM, with 0 factors as the reference group). Results Greater pre-pregnancy adiposity, pregnancy weight gain and GDM were independently associated with increased risk of giving birth to an LGA infant across all races (except GDM among non-Hispanic whites), in both underweight and normal-weight women. Among non-Hispanic white, non-Hispanic black and Hispanic women, the threefactor joint effect was associated with substantially increased odds of LGA (OR [95\% CI] 11.27 [8.40, 15.11], 7.09 [4.81, 10.45] and 10.19 [6.84, 15.19], respectively). However, for Asian women the joint effect of all three factors (OR [95\% CI] $5.14[2.11,12.50]$ ) was approximately the same as any of the two factors.

Conclusions/interpretation GDM, pre-pregnancy obesity and excessive pregnancy weight gain were jointly associated with elevated risk of giving birth to an LGA infant and the effects varied by race. This suggests that those involved in public health efforts aimed at preventing LGA deliveries should consider variations in racial groups when devising effective strategies.

Keywords Adiposity $\cdot$ Fetal macrosomia $\cdot$ Gestational diabetes $\cdot$ Pregnancy $\cdot$ Weight gain
Abbreviations
ACOG American College of Obstetricians and Gynecologists
CSL Consortium on Safe Labor
GDM Gestational diabetes mellitus
GEE Generalised estimating equations
IOM Institute of Medicine
LGA Large for gestational age
PI Pacific islander 


\section{Introduction}

The escalating rate of childhood obesity has become a serious clinical and public health concern worldwide [1] within certain ethnic groups being disproportionately affected [2-4]. Growing evidence suggests that the intrauterine environment may play a critical role in the development of obesity and other metabolic outcomes. In efforts to control childhood obesity, it has become increasingly important to identify major preconception- and pregnancy-related risk factors associated with excessive fetal growth as this may represent the true starting point of a life-long propensity to obesity [5].

Maternal gestational diabetes (GDM) and hyperglycaemia in pregnancy have long been related to excessive fetal growth [6-9]. Maternal obesity before pregnancy and excessive weight gain during pregnancy are additional, potentially modifiable, independent risk factors of excessive fetal growth [10] and often occur in conjunction with GDM or hyperglycaemia in pregnancy. It is therefore important to evaluate the association of pre-pregnancy obesity and weight gain during pregnancy in the context of the two other major risk factors for excess fetal growth. Moreover, as the prevalence of both GDM and childhood obesity varies by race/ethnicity, the effects of these factors on fetal growth may differ accordingly. Indeed, data from a recent study of 140,128 births among nonHispanic white women and 82,492 non-Hispanic black women in South Carolina suggested that the combined effects of pre-pregnancy obesity and GDM differed between these two groups, with a stronger combined effect of GDM and BMI in black women than in white women [11]. However, given the small number of GDM cases, the interplay of the three major risk factors has not been systematically investigated across multiple races (such as Asians and Hispanics_-populations at high risk of GDM).

Using data from the Consortium on Safe Labor (CSL), a large, representative cohort of pregnancies in USA, our objective in this study was to identify subgroups of the population that are particularly susceptible to the adverse impact of maternal hyperglycaemia in pregnancy and to examine the joint and independent effects of GDM, maternal pre-pregnancy BMI and gestational weight gain on excessive fetal growth across four race/ethnicity groups (i.e. non-Hispanic white, non-Hispanic black, Hispanic and Asian/Pacific Islander [PI]). Such data may be pivotal in developing targeted and efficient strategies for the life-long battle with obesity.

\section{Methods}

Data acquisition The CSL was a study initiated by the Eunice Kennedy Shriver National Institute of Child Health and Human Development, National Institutes of Health, in collaboration with 12 institutions across the USA and has been described in detail elsewhere [12]. In brief, this was a retrospective observational study of births between 2002 and 2008 from 19 hospitals, representing nine American College of Obstetricians and Gynecologists (ACOG) districts. In total, data from 228,562 deliveries were abstracted, including demographic data, medical history, and labour and delivery information in addition to obstetrical, postpartum and neonatal outcomes. Data were abstracted from patient electronic medical records. Maternal and newborn discharge International Classification of Diseases (ICD)-9 codes (www.icd9data.com/2007/Volume1/240-279/250-259/250/ default.htm) were also collected for each delivery. The data coordinating centre received electronic data from each study site and mapped the data to pre-defined variables. Data inquiries, cleaning and logic checking were performed. Validation studies determined that the constructed database was a highly valid representation of the electronic medical records [12].

Population Exclusion criteria for the analyses reported here include: multiple gestation pregnancies $(n=5,059)$; missing data on major exposures, outcomes and covariates of the study, including pre-pregnancy BMI $(n=58,618)$, weight at admission to the hospital prior to the birth $(n=4,384)$, GDM $(n=25,600)$ and birthweight $(n=2,340)$ and medical history of type 2 diabetes $(n=4,213)$. Moreover, we excluded pregnancies less than 24 weeks $(n=1,760)$ and one site $(n=$ 20,603) was excluded due to suspected under-reporting of GDM where the prevalence of $<1 \%$ was substantially lower than that expected in the general US population. The final analytical population was composed of 105,985 pregnancies.

Obesity was defined as pre-pregnancy BMI $>25 \mathrm{~kg} / \mathrm{m}^{2}$. High pregnancy weight gain was defined using two methods. First, we used the 2009 Institute of Medicine (IOM) guidelines for weight gain in pregnancy, which contain various recommendations based on pre-pregnancy BMI [13]. Second, we defined high pregnancy weight gain as greater than the median weight gain within the CSL cohort. The results were not appreciably different and we provide results for the data-driven method defining weight gain as greater than the median. Determination of GDM status was recorded in the medical records. Birthweight was captured from labour and delivery records and macrosomia was defined using two common clinical cut-points: $>4,000 \mathrm{~g}$ and $>4,500 \mathrm{~g}$. Infant size was defined as LGA if the infants were $>90$ th percentile birthweight at each gestational age for each race and fetal sex.

Statistical analyses Baseline characteristics of women were compared across categories of offspring size, including LGA, macrosomia (defined as both $>4,000 \mathrm{~g}$ and $>4,500 \mathrm{~g}$ ) and 
normal weight $(2,700-4,000 \mathrm{~g})$, and by race/ethnicity. Proportions were provided for categorical variables and means and SDs for continuous variables. Characteristics were also compared between subjects missing and not missing data for important covariates. Generalised estimating equations (GEE) were used to estimate the OR for giving birth to a macrosomic or LGA infant for women with GDM vs women without GDM to account for correlation inherent in multiple pregnancies experienced by some women. Generalised linear models with GEE were used to estimate the association between GDM and birthweight. Models were adjusted for potential confounding factors, which were a priori risk factors for GDM and excess fetal growth and not in the causal pathway. To determine whether the association between GDM and infant size varied across categories of BMI $(<18.5, \geq 18.5$ and $<25, \geq 25$ and $<30, \geq 30 \mathrm{~kg} / \mathrm{m}^{2}$ ), pregnancy weight gain (quartiles) and race (non-Hispanic white, non-Hispanic black, Hispanic, Asian/PI, multi-ethnic/other), effect modification was evaluated using multiplicative interaction terms and stratification. In addition, joint effects modelling considering one, two or three factors, with 0 factors as the reference group, was employed. We restricted the interaction and joint effect analyses to LGA, accounting for race, fetal sex and gestational age, based on our objective of evaluating differences by race and ethnicity. All statistical analyses were performed with SAS software version 9.3 (SAS Institute, Cary, NC, USA).

\section{Results}

After exclusions, the analytical population was composed of 105,985 pregnancies of which 10,689 neonates were LGA, 7,718 were macrosomic with a birthweight $>4,000 \mathrm{~g}$ and 964 were macrosomic with a birthweight $>4,500 \mathrm{~g}$ (Table 1). In general, macrosomic and LGA infants were more likely than normal-weight infants to be born from pregnancies affected by GDM and from women who had a history of a macrosomic baby. Birthweight, regardless of characterisation, was linearly associated with pre-pregnancy BMI, with the highest proportion of large babies born to women whose pre-pregnancy BMI was $\geq 30 \mathrm{~kg} / \mathrm{m}^{2}$. Similarly, increasing quartiles of weight gain (defined as admission weight minus pre-pregnancy weight in kilograms) were linearly associated with the proportion of infants born LGA or macrosomic, regardless of definition. Asian/PI women had the highest proportion of babies born LGA (12.13\%) and white women had the smallest proportion (9.74\%) (Table 2); Hispanic women had the highest proportion of macrosomic infants (birthweight of either $>4,000 \mathrm{~g}$ or $>4,500 \mathrm{~g}$ ). Moreover, compared with women of other race/ethnicity, Asian/PI women were less likely to be overweight or obese before pregnancy and were less likely to exceed weight gain during pregnancy as recommended by the
2009 IOM guidelines. Of note, the prevalence of both GDM and LGA infants was highest for Asian/PI women and lowest for non-Hispanic white women.

After adjustment for maternal age, weight gain during pregnancy, pre-pregnancy BMI, type of medical insurance, history of macrosomia and parity, GDM was associated with an approximately twofold increased risk for delivering an LGA baby (OR 2.02 [95\% CI 1.86, 2.19]). The multivariate adjusted risk estimate (adjusting for the same covariates) for the association between GDM and macrosomia defined as $>4,000 \mathrm{~g}$ was $1.48(95 \%$ CI $1.34,1.64)$ and for macrosomia defined as $>4,500 \mathrm{~g}$ the OR was $2.59(95 \%$ CI $2.11,3.18)$ (Table 3). Adding height to the models did not change the OR appreciably $(<10 \%)$. Finally, adjusting for covariates, having GDM was associated with a $49.18 \mathrm{~g}$ increase in continuous birthweight (95\% CI 39.10, 67.26) (data not shown).

The association between GDM and LGA delivery varied by category of pre-pregnancy BMI ( $p$ value for interaction $<$ 0.0001 ), being strongest among obese women and weakest among women in the normal BMI range; the OR $(95 \% \mathrm{CI})$ was $2.10(1.87,2.37), 1.77(1.52,2.06)$ and $1.57(1.31$, 1.89) for obese, overweight and normal-weight women, respectively (data not shown). There was also a significant three-way interaction between GDM, pre-pregnancy BMI and race $(p$ value for interaction=0.001). Except for nonHispanic white women, all normal-weight women with GDM demonstrated a significant increased risk for delivery of an LGA infant. In addition, underweight women tended to also have an increased risk across races, though the stratified results were not significant. The association between GDM and an LGA delivery also varied across categories of pregnancy weight gain ( $p$ value for interaction $<0.0001$ (data not shown).

Table 4 shows the independent and joint effects of prepregnancy obesity, pregnancy weight gain and GDM on the risk for LGA delivery in comparison to women without any of these risk factors separately for each racial group. Across all races, having GDM, obesity and high pregnancy weight gain was significantly and strongly associated with having an LGA infant although the effect estimates varied across races; OR (95\% CI) was 11.27 (8.40, 15.11), 7.09 (4.81, $10.45), 10.19(6.84,15.19)$ and $5.14(2.11,12.50)$ for nonHispanic white, non-Hispanic black, Hispanic and Asian/PI women, respectively. With the exception of GDM in nonHispanic white women, the presence of a single factor was enough to significantly increase the risk for LGA delivery and the presence of two factors appreciably increased the risk compared with women having no risk factors. In nonHispanic white women, GDM was not significantly associated with LGA delivery unless it occurred in combination with either pre-pregnancy obesity or excessive weight gain in pregnancy. The presence of two factors appreciably increased the risk compared with women having no risk 
Table 1 Baseline maternal and infant characteristics by measures of excess fetal growth including LGA and macrosomia among 105,985 pregnancies

\begin{tabular}{|c|c|c|c|c|}
\hline Characteristic & $\mathrm{LGA}^{\mathrm{a}}$ & Macrosomia $(>4,000 \mathrm{~g})$ & Macrosomia $(>4,500 \mathrm{~g})$ & Normal weight $(2,700-4,000 \mathrm{~g})$ \\
\hline$n$ & 10,689 & 7,718 & 964 & 85,564 \\
\hline \multicolumn{5}{|l|}{ Maternal characteristic } \\
\hline GDM & $891(8.3)$ & $511(6.6)$ & $125(13.0)$ & $3,326(3.9)$ \\
\hline History of macrosomia & $784(7.3)$ & $612(7.9)$ & $152(15.8)$ & $1,626(1.9)$ \\
\hline \multicolumn{5}{|l|}{$\operatorname{BMI}\left(\mathrm{kg} / \mathrm{m}^{2}\right)$} \\
\hline$<18.5$ & $230(2.2)$ & $160(2.1)$ & $11(1.1)$ & $4,157(4.9)$ \\
\hline$\geq 18.5$ and $<25$ & $4,344(40.6)$ & $3,271(42.4)$ & $302(31.3)$ & $46,136(53.9)$ \\
\hline$\geq 25$ and $<30$ & $3,006(28.1)$ & $2,164(28.0)$ & $297(30.8)$ & $19,630(22.9)$ \\
\hline$\geq 30$ & $3,109(29.1)$ & $2,123(27.5)$ & $354(36.7)$ & $15,641(18.3)$ \\
\hline \multicolumn{5}{|l|}{ Race } \\
\hline Non-Hispanic white & $5,611(52.5)$ & $4,505(58.4)$ & $508(52.7)$ & $47,569(55.6)$ \\
\hline Non-Hispanic black & $1,884(17.6)$ & $872(11.3)$ & $130(13.5)$ & $13,750(16.1)$ \\
\hline Hispanic & $2,180(20.4)$ & $1,668(21.6)$ & $241(25.0)$ & $16,281(19.0)$ \\
\hline Asian/PI & $352(3.3)$ & $160(2.1)$ & $18(1.9)$ & $2,422(2.8)$ \\
\hline Multi/other/unknown & $662(6.2)$ & $513(6.7)$ & $67(7.0)$ & $5,542(6.5)$ \\
\hline Age (years) & $28.65(5.8)$ & $28.51(5.7)$ & $29.39(20.0)$ & $27.22(5.8)$ \\
\hline \multicolumn{5}{|l|}{ Parity } \\
\hline 0 & $3,174(29.7)$ & $2,534(32.8)$ & $289(30.0)$ & $32,690(38.2)$ \\
\hline 1 & $3,439(32.2)$ & $2,404(31.2)$ & $323(33.5)$ & $25,885(30.3)$ \\
\hline 2 & $2,128(19.9)$ & $1,424(18.5)$ & $159(16.5)$ & $15,000(17.5)$ \\
\hline 3 or more & $1,948(18.2)$ & $1,356(17.6)$ & $193(20.0)$ & $11,989(14.0)$ \\
\hline \multicolumn{5}{|l|}{ Insurance } \\
\hline Private & $6,266(58.6)$ & $4,763(61.7)$ & $564(58.5)$ & $50,914(59.5)$ \\
\hline Public & $3,420(32.0)$ & $2,333(30.2)$ & $331(34.3)$ & $26,102(30.5)$ \\
\hline Self-pay & $106(1.0)$ & $75(1.0)$ & $13(1.4)$ & $833(1.0)$ \\
\hline Other/unknown & $897(8.4)$ & $547(7.1)$ & $56(5.8)$ & $7,715(9.0)$ \\
\hline Weight gain in pregnancy $(\mathrm{kg})$ & $16.23(7.5)$ & $16.86(7.2)$ & $17.98(8.4)$ & $14.17(6.6)$ \\
\hline \multicolumn{5}{|c|}{ Weight gain in pregnancy vs IOM recommendations } \\
\hline Exceeded & $8,276(66.9)$ & $6,353(70.3)$ & $893(79.4)$ & $47,181(47.7)$ \\
\hline Met & $2,824(22.8)$ & $1,992(22.0)$ & $166(14.8)$ & $32,988(33.3)$ \\
\hline Below & $1,272(10.3)$ & $696(7.7)$ & $66(5.9)$ & $18,814(19.0)$ \\
\hline Quartile $1(<10.43 \mathrm{~kg})$ & $1,978(7.2)$ & $1,179(4,3)$ & $148(0.5)$ & $20,950(76.3)$ \\
\hline Quartile $2(10.43-14.06$ kg) & $2,032(7.7)$ & $1,384(5.3)$ & $156(0.6)$ & $21,727(82.7)$ \\
\hline Quartile 3 (14.07-18.14 kg) & $2,560(10.1)$ & $1,952(7.7)$ & $175(0.7)$ & $21,182(83.5)$ \\
\hline Quartile 4 (>18.14 kg) & $4,119(15.3)$ & $3,203(11.9)$ & $485(1.8)$ & $21,705(80.7)$ \\
\hline \multicolumn{5}{|l|}{ Infant characteristic } \\
\hline Gestational age (weeks) & $38.60(2.2)$ & $39.64(1.04$ & $39.67(1.1)$ & $39.01(1.2)$ \\
\hline Birthweight (g) & $4,026.7(482.8)$ & $4,245.5(229.4)$ & $4,719.0(214.7)$ & $(321.9)$ \\
\hline
\end{tabular}

Continuous variables are expressed as means (SD) and categorical variables are expressed as $n(\%)$

${ }^{a}$ LGA is defined as $>90$ th percentile birthweight for gestational age, race and fetal sex

factors, although the combination of effects varied across races. For Asian women, when considering multiple risk factors, GDM was associated with the strongest effect. For example, having GDM in combination with either high weight gain or obesity was associated with around a fivefold increased risk for LGA delivery, which did not change appreciably when considering all three factors together. However, for Hispanic and non-Hispanic white women, the association with LGA delivery more than doubled when comparing three risk factors with two. For non-Hispanic black women, the smallest effect alone, and in combination with other factors, was pre-pregnancy obesity (OR 1.87 
Table 2 Baseline maternal and infant characteristics by race/ethnicity

\begin{tabular}{|c|c|c|c|c|c|}
\hline Characteristic & Non-Hispanic white & Non-Hispanic black & Hispanic & Asian/PI & Multi/other \\
\hline$n$ & 66,496 & 20,578 & 23,397 & 4,405 & 7,316 \\
\hline \multicolumn{6}{|l|}{ Maternal characteristic } \\
\hline $\mathrm{LGA}^{\mathrm{a}}$ & $6,477(9.7)$ & $2,185(10.6)$ & $2,487(10.6)$ & $5,209(12.0)$ & $694(9.5)$ \\
\hline GDM & $1,942(3.4)$ & $620(3.4)$ & $1,209(6.0)$ & $260(9.0)$ & $320(4.6)$ \\
\hline History of macrosomia & $1,309(2.0)$ & $351(1.7)$ & $507(2.2)$ & $39(0.9)$ & $120(1.6)$ \\
\hline BMI $\left(\mathrm{kg} / \mathrm{m}^{2}\right)$ & $24.6(5.7)$ & $27.4(7.3)$ & $25.5(5.6)$ & $22.9(4.9)$ & $25.0(5.8)$ \\
\hline$<18.5$ & $3,898(5.9)$ & $805(3.9)$ & $992(4.2)$ & $516(11.7)$ & $442(6.04)$ \\
\hline$\geq 18.5$ and $<25$ & $39,375(59.2)$ & $8,564(41.6)$ & $11,988(51.2)$ & $2,879(65.4)$ & $3,982(54.4)$ \\
\hline$\geq 25$ and $<30$ & $13,323(20.0)$ & $5,368(26.1)$ & $6,262(26.8)$ & $624(14.2)$ & $1,720(23.5)$ \\
\hline$\geq 30$ & 9,900 (14.9) & $5,841(28.4)$ & $4,155(17.8)$ & $386(8.8)$ & $1,172(16.0)$ \\
\hline Maternal age (years) & $28.5(5.7)$ & $26.1(6.5)$ & $27.3(6.3)$ & $30.4(5.3)$ & $27.2(6.2)$ \\
\hline \multicolumn{6}{|l|}{ Parity } \\
\hline 0 & $26,389(39.7)$ & $8,142(39.6)$ & $8,828(37.7)$ & $2,206(50.1)$ & $3,493(47.7)$ \\
\hline 1 & $20,191(30.4)$ & $5,792(28.2)$ & $7,405(31.7)$ & $1,425(32.4)$ & $2,052(28.1)$ \\
\hline 2 & $11,028(16.6)$ & $3,498(17.0)$ & $4,234(18.1)$ & 467 (10.6) & $1,001(13.7)$ \\
\hline 3 or more & $8,888(13.4)$ & $3,146(15.3)$ & $2,930(12.5)$ & $307(7.0)$ & $770(10.5)$ \\
\hline \multicolumn{6}{|l|}{ Insurance } \\
\hline Private & $49,198(74.0)$ & $6,253(30.4)$ & $5,409(23.1)$ & $2,674(60.7)$ & $3,706(50.7)$ \\
\hline Public & $10,955(16.5)$ & $9,538(46.4)$ & $10,459(44.7)$ & $581(13.2)$ & $2,163(29.6)$ \\
\hline Self-pay & $296(0.5)$ & $301(1.5)$ & $322(1.4)$ & $57(1.3)$ & $91(1.2)$ \\
\hline Other/unknown & $6,047(9.1)$ & $4,486(21.8)$ & $7,207(30.8)$ & $1,093(24.8)$ & $1,356(18.5)$ \\
\hline Weight gain in pregnancy $(\mathrm{kg})$ & $14.5(6.3)$ & $14.0(7.9)$ & $13.5(6.5)$ & $14.3(5.7)$ & $14.2(6.6)$ \\
\hline Quartile $1(<10.43 \mathrm{~kg})$ & $14,126(21.2)$ & $6,277(30.5)$ & $7,083(30.3)$ & $891(20.2)$ & $1,879(25.7)$ \\
\hline Quartile $2(10.43-14.06 \mathrm{~kg})$ & $17,161(25.8)$ & $4,334(21.1)$ & $5,707(24.4)$ & $1,212(27.5)$ & $1,819(24.9)$ \\
\hline Quartile 3 (14.07-18.14 kg) & $18,033(27.1)$ & $4,215(20.5)$ & $5,304(22.7)$ & $1,339(30.4)$ & $1,707(23.3)$ \\
\hline Quartile 4 (>18.14 kg) & $17,176(25.8)$ & $5,752(28.0)$ & $5,303(22.7)$ & $963(21.9)$ & $1,911(26.1)$ \\
\hline \multicolumn{6}{|c|}{ Weight gain in pregnancy vs IOM recommendation } \\
\hline Exceeded & $31,450(47.3)$ & $10,711(52.1)$ & $10,658(45.6)$ & $1,760(40.0)$ & $3,460(47.3)$ \\
\hline Met & $22,767(34.2)$ & $5,263(25.6)$ & $7,337(31.4)$ & $1,700(38.6)$ & $2,354(32.2)$ \\
\hline Below & $12,279(18.5)$ & $4,604(22.4)$ & $5,402(23.1)$ & $945(21.5)$ & $1,502(20.5)$ \\
\hline \multicolumn{6}{|l|}{ Infant characteristic } \\
\hline Gestational age (weeks) & $38.8(1.8)$ & $38.4(2.5)$ & $38.7(2.1)$ & $38.9(1.6)$ & $38.6(2.3)$ \\
\hline Birthweight $(\mathrm{g})$ & $3,326.7(529.5)$ & $3,120.6(632.9)$ & $3,287.2(577.8)$ & $3,252.9(495.8)$ & $(600.8)$ \\
\hline
\end{tabular}

Continuous variables are expressed as means (SD) and categorical variables are expressed as number (\%)

${ }^{a}$ LGA is defined as $>90$ th percentile birthweight for gestational age, race and fetal sex

[95\% CI, 1.59, 2.18]), while GDM and high weight gain were both important risk factors with the two-factor joint effect, stronger than in any other race (OR 7.59 [95\% CI, $5.34,10.80])$.

\section{Discussion}

In this large multi-race/ethnicity cohort in the USA, we found that not only are GDM, pre-pregnancy adiposity and pregnancy weight gain individually associated with excess fetal growth, but also their combined effects lead to substantially increased odds for having a baby born LGA. The influence of varying combinations of these factors differed by race with the effect of all three factors ranging from five- to over 11-fold across races. Specifically, either alone or in combination with either pre-pregnancy obesity or high weight gain, GDM in Asian women was significantly associated with increased risk of LGA delivery; however, the presence of all three factors together did not increase the risk further. Contrary to that finding, compared with having only two factors, the presence of all three factors more than doubled the risk for LGA delivery in both Hispanic and nonHispanic white women. Of note, among non-Hispanic white 
Table 3 OR $(95 \% \mathrm{CI})$ describing the association between GDM, covariates and multiple characterisations of offspring size at birth including LGA and macrosomia
${ }^{a}$ LGA, large for gestational age, race and sex

${ }^{\mathrm{b}}$ All variables in multivariate model are mutually adjusted ref, reference

\begin{tabular}{|c|c|c|c|}
\hline Independent variable & $\mathrm{LGA}^{\mathrm{a}}$ & Macrosomia $(>4,000 \mathrm{~g})$ & $\operatorname{Macrosomia}(>4,500 \mathrm{~g})$ \\
\hline GDM & $2.02(1.86,2.19)^{\mathrm{b}}$ & $1.48(1.34,1.64)$ & $2.59(2.11,3.18)$ \\
\hline \multicolumn{4}{|l|}{ Maternal age (years) } \\
\hline$<25$ & 1.00 (ref) & 1.00 (ref) & 1.00 (ref) \\
\hline $25-29.9$ & $1.52(1.44,1.61)$ & $1.57(1.40,1.76)$ & $1.94(1.35,2.79)$ \\
\hline$\geq 30$ & $2.20(2.08,2.33)$ & $1.86(1.63,2.12)$ & $2.59(1.74,3.86)$ \\
\hline \multicolumn{4}{|l|}{ Type of insurance } \\
\hline Private & 1.00 (ref) & 1.00 (ref) & 1.00 (ref) \\
\hline Public & $0.92(0.88,0.97)$ & $0.89(0.84,0.94)$ & $0.93(0.80,1.07)$ \\
\hline Self-pay & $0.97(0.89,1.06)$ & $0.92(0.73,1.17)$ & $1.18(0.67,2.07)$ \\
\hline Other & $0.97(0.89,1.06)$ & $0.84(0.75,0.93)$ & $0.69(0.52,0.93)$ \\
\hline History of macrosomia & $3.41(3.10,3.75)$ & $3.62(3.26,4.01)$ & $5.70(4.66,6.97)$ \\
\hline \multicolumn{4}{|l|}{ Parity } \\
\hline 0 & 1.00 (ref) & 1.00 (ref) & 1.00 (ref) \\
\hline 1 & $1.40(1.33,1.48)$ & $1.20(1.13,1.27)$ & $1.27(1.08,1.51)$ \\
\hline 2 & $1.42(1.33,1.51)$ & $1.16(1.08,1.25)$ & $0.94(90.77,1.16)$ \\
\hline 3 or more & $1.51(1.41,1.62)$ & $1.31(1.22,1.42)$ & $1.25(1.02,1.53)$ \\
\hline \multicolumn{4}{|l|}{ Race } \\
\hline Non-Hispanic white & 1.00 (ref) & 1.00 (ref) & 1.00 (ref) \\
\hline Non-Hispanic black & $0.97(0.92,1.04)$ & $0.54(0.50,0.59)$ & $0.67(0.54,0.83)$ \\
\hline Hispanic & $1.14(1.07,1.21)$ & $1.15(1.08,1.23)$ & $1.41(1.19,1.66)$ \\
\hline Asian/PI & $1.39(1.23,1.56)$ & $0.74(0.63,0.88)$ & $0.73(0.45,1.18)$ \\
\hline Multi-ethnic/other & $1.02(0.93,1.12)$ & $1.00(0.91,1.11)$ & $1.13(0.87,1.47)$ \\
\hline \multicolumn{4}{|c|}{ Weight gain in pregnancy vs IOM recommendations } \\
\hline Met or below & 1.00 (ref) & 1.00 (ref) & 1.00 (ref) \\
\hline Exceeded & $1.42(1.33,1.51)$ & $1.55(1.44,1.66)$ & $1.93(1.59,2.34)$ \\
\hline
\end{tabular}

Table 4 Multivariate ${ }^{\mathrm{a}}$ adjusted OR $(95 \% \mathrm{CI})$ of the association with delivery of an LGA infant for varying combinations of risk factors

\begin{tabular}{|c|c|c|c|c|c|c|c|c|}
\hline \multirow[t]{2}{*}{ No. of risk factors } & \multicolumn{2}{|l|}{ Non-Hispanic white } & \multicolumn{2}{|l|}{ Non-Hispanic black } & \multicolumn{2}{|l|}{ Hispanic } & \multicolumn{2}{|l|}{ Asian/PI } \\
\hline & OR $(95 \% \mathrm{CI})$ & $n$ & OR $(95 \% \mathrm{CI})$ & $n$ & OR $(95 \% \mathrm{CI})$ & $n$ & OR $(95 \% \mathrm{CI})$ & $n$ \\
\hline 0 factors $^{\mathrm{b}}$ & 1.00 (ref) & 20,771 & 1.00 (ref) & 6,011 & 1.00 (ref) & 8,197 & 1.00 (ref) & 1,094 \\
\hline \multicolumn{9}{|l|}{1 factor } \\
\hline GDM & $1.23(0.90,1.66)$ & 668 & $2.32(1.34,4.00)$ & 115 & $1.83(1.37,2.45)$ & 475 & $2.62(1.51,4.53)$ & 134 \\
\hline High weight gain ${ }^{\mathrm{c}}$ & $2.27(2.11,2.44)$ & 26,186 & $1.95(1.71,2.24)$ & 6,546 & $2.25(2.00,2.52)$ & 7,653 & $2.77(2.04,3.74)$ & 1,247 \\
\hline Obesity & $2.17(1.96,2.40)$ & 5,885 & $1.87(1.59,2.18)$ & 3,226 & $1.81(1.54,2.13)$ & 2,256 & $2.65(1.66,4.23)$ & 193 \\
\hline \multicolumn{9}{|l|}{2 factors } \\
\hline $\begin{array}{l}\text { GDM and high } \\
\text { weight gain }\end{array}$ & $3.44(2.67,4.43)$ & 467 & $7.59(5.34,10.80)$ & 157 & $4.68(3.55,6.18)$ & 305 & $4.42(2.32,8.39)$ & 69 \\
\hline $\begin{array}{l}\text { Obesity and high } \\
\text { weight gain }\end{array}$ & $4.68(4.18,5.24)$ & 2,805 & $3.07(2.60,3.62)$ & 1,831 & $4.42(3.70,5.28)$ & 999 & $3.83(2.20,6.67)$ & 107 \\
\hline GDM and obesity & $3.69(2.98,4.58)$ & 611 & $4.08(2.94,5.66)$ & 215 & $4.03(3.04,5.34)$ & 314 & $5.81(2.54,13.26)$ & 35 \\
\hline $\begin{array}{l}3 \text { factors (GDM, } \\
\text { obesity and high } \\
\text { weight gain) }\end{array}$ & $11.27(8.40,15.11)$ & 196 & $7.09(4.81,10.45)$ & 133 & $10.19(6.84,15.19)$ & 115 & $5.14(2.11,12.50)$ & 22 \\
\hline
\end{tabular}

${ }^{a}$ Multivariate models adjusted for maternal age, parity, type of insurance and history of macrosomia

${ }^{\mathrm{b}}$ All groups are compared with the reference (low-risk) group, which includes non-obese women without high weight gain and without GDM

${ }^{\mathrm{c}}$ High weight gain is defined as greater than the cohort median 
women, GDM was not significantly associated with LGA delivery unless it was present in combination with either pre-pregnancy obesity or excessive weight gain in pregnancy. Physiological differences in glucose metabolism and/or fat storage may account for some of these differences and further research to understand these differences may help elucidate the underlying pathology of GDM and related metabolic impairments.

The association of GDM, maternal obesity before pregnancy and weight gain during pregnancy with excess fetal growth has been widely studied individually $[6,14-16]$. As each of these risk factors often occur in conjunction with the others, it is important to evaluate the association of each factor in the context of the two other major risk factors for excess fetal growth. For example, a recent retrospective study of 9,835 women found an increased risk for delivery of an LGA baby among overweight and obese women that was compounded when accompanied by GDM and the effects are additive [17]. However, as the prevalence of both GDM and childhood obesity varies by race/ethnicity the effects of these factors on fetal growth may differ by race/ethnicity. The race-specific joint effects of three major risk factors have previously not been comprehensively analysed. Our results are consistent with a recent comparison of two races in South Carolina which showed that the impact of GDM on birthweight was stronger among non-Hispanic black than non-Hispanic white women [11]. However, this study was limited to two races and evaluated the impact of maternal BMI at delivery, but did not consider either pre-pregnancy BMI or weight gain during pregnancy. Using the CSL, a nationally representative cohort, we were able to identify additional racial differences, as well as estimate the contribution made by all three risk factors for LGA delivery, including pre-pregnancy BMI, pregnancy weight gain and GDM.

Discrepancies in earlier results could be due to differences in the study populations (e.g. in terms of race and ethnicity). Rates of GDM differ by race, with black, Hispanic [18] and Asian [19] populations having higher rates compared with non-Hispanic whites. In addition, previous studies have identified racial variation in the risk for macrosomia in infants born to women with GDM [20-23]. For example, comparing varying races in a Hawaiian population, Silva et al identified greater risk for delivery of infants with macrosomia among native Hawaiian/PI and Filipino women with GDM compared with Japanese, Chinese and white women [22]. Interestingly, the rate of GDM was second lowest (3.6\%) among native Hawaiian/PIs (compared with $4.8 \%$ overall and $2.3 \%$ for white women), despite them having the highest mean BMI, greater than four units higher than the closest group [22]. In addition, previous studies identified Latino women as being at higher risk of delivering infants with macrosomia resulting from GDM in comparison with African-American women [21], and found that African-American women were at higher risk than non-Hispanic white women [20].

Variations in the influence of GDM and weight characteristics on macrosomia by race may be due to differences in metabolism exemplified by variation in body composition and/or genetics. For example, although Asian and PI populations display high levels of GDM, BMI is not a major predictor of risk compared with other ethnic groups [24] and the mean BMI in the present study was appreciably lower for Asian/PI women compared with other races. However, compared with the white population, at lower BMI Asians have a higher percentage of body fat [25]. We recently identified a significant association between height and GDM that was strongest among Asian women (J. Brite, E. Shiroma, K. Bowers, E. Yeung, S. K. Laughon, U. Grewal, C. Zhang, unpublished results). Regional fat distribution may also vary between African-American and white women [26]; one study identified larger subcutaneous adipose tissue depots, adjusted for total fat, among AfricanAmerican women [26].

One limitation of the present study is the reduced size of the analytical population due to missing data on prepregnancy BMI, a major risk factor for excess fetal growth. A comparison of maternal and infant characteristics between subjects missing and not missing data for pre-pregnancy BMI revealed few differences. The subjects who were and were not missing data on BMI did not differ appreciably by maternal age (27.7 vs 27.2 years), pre-pregnancy weight (68.1 vs $68.2 \mathrm{~kg}$ ) or hospital admission weight (83.4 vs $82.2 \mathrm{~kg}$ ), nor did their infants differ by birthweight $(3,236.5$ vs $3,266.7$ g.) or gestational age $(38.5$ vs 38.6 weeks). They did differ slightly by race, with fewer non-Hispanic white $(50.0 \%$ vs $54.1 \%)$ and Hispanic $(11.6 \%$ vs $18.9 \%)$ and more non-Hispanic black (23.4\% vs $17.7 \%)$ and Asian/PI (5.0\% vs 2.8\%) women missing data on prepregnancy BMI. This may have affected our estimated contribution from BMI, especially if data from individuals with high BMI were more likely to be missing. As a sensitivity analysis, we imputed BMI using multiple imputation. The results did not differ appreciably from the main study results. Another limitation of this study is the classification of race/ethnicity in CSL. Specifically, Asian and PI populations were combined, although a prior study comparing Asians and PIs found varying risks for delivery of infants with macrosomia among native Hawaiian, PI and Filipino women with GDM compared with Japanese, Chinese and white women [22]. Additional differences exist between East Asians. For example, a recent study found differences in the association between GDM and delivery of infants with macrosomia for Cambodian and Laotian women compared with Japanese women [23]. Finally, we 
were missing data on potential covariates of interest, such as additional variables representing socioeconomic status, prenatal care, physical activity, diet and diabetes control, which could help explain race/ethnicity disparities in the association.

There are several strengths to our study, including the large cohort of pregnancies, representative of the US population. In addition, our analyses were strengthened by defining size at birth as LGA for each race and fetal sex. While clear cut-offs for macrosomia, such as $>4,000$ and $>4,500 \mathrm{~g}$, may be useful in a clinical setting because of their simplicity, they may underestimate the number of babies with excessive fetal growth due to inherent racial differences in body size. For example, if Asian women deliver smaller babies compared with other races, it will take a greater excess of growth to reach a standard cut-off such as 4,000 g. Additionally, males tend to be bigger babies than females. Therefore, by calculating size for gestational age, fetal sex and race, we were more likely to capture an appropriate measure of excess size.

In this large multi-race/ethnicity cohort in the USA, we observed that GDM was significantly associated with risk of women giving birth to macrosomic and LGA infants, even among normal-weight and underweight women. More importantly, this association was significantly modified by prepregnancy BMI, pregnancy weight gain and race. The joint effects of pre-pregnancy obesity, weight gain during pregnancy and GDM on fetal growth also varied by race/ethnicity. These results suggest that a woman's race may be an important consideration when developing prevention strategies for excess fetal growth in women with GDM.

Acknowledgements The data included in this paper were obtained from the CSL, which was supported by the Intramural Research Program of the Eunice Kennedy Shriver National Institute of Child Health and Human Development, National Institutes of Health, through Contract No. HHSN267200603425C. Institutions involved in the CSL include, in alphabetical order: Baystate Medical Center, Springfield, MA, USA; Cedars-Sinai Medical Center Burnes Allen Research Center, Los Angeles, CA, USA; Christiana Care Health System, Newark, DE, USA; Georgetown University Hospital, MedStar Health, Washington, DC, USA; Indiana University Clarian Health, Indianapolis, IN, USA; Intermountain Healthcare and the University of Utah, Salt Lake City, UT, USA; Maimonides Medical Center, Brooklyn, NY, USA; MetroHealth Medical Center, Cleveland, OH, USA; Summa Health System, Akron City Hospital, Akron, OH, USA; The EMMES Corporation, Rockville MD, USA (data coordinating centre); University of Illinois at Chicago, Chicago, IL, USA; University of Miami, Miami, FL, USA; and University of Texas Health Science Center at Houston, Houston, TX, USA. The named authors alone are responsible for the views expressed in this manuscript, which do not necessarily represent the decisions or the stated policy of the National Institute of Child Health and Human Development.

Funding K. Bowers, S. K. Laughon, M. Kiely, Z. Chen and C. Zhang were supported by the Intramural Research Program of the Eunice
Kennedy Shriver National Institute of Child Health and Human Development, National Institutes of Health.

Duality of interest The authors declare that there is no duality of interest associated with this manuscript.

Contribution statement $\mathrm{KB}$ was responsible for data analyses and drafting the manuscript. ZC analysed data and revised the manuscript. SKL was responsible for conception of the study and revision of the manuscript. MK and JB were involved in interpretation of the data and revision of the manuscript and $\mathrm{CZ}$ was responsible for conception of the study and revision of the manuscript. All authors approved the final version to be published.

\section{References}

1. Wang Y, Lobstein T (2006) Worldwide trends in childhood overweight and obesity. Int J Pediatr Obes 1:11-25

2. Wang Y, Tussing L (2004) Culturally appropriate approaches are needed to reduce ethnic disparity in childhood obesity. J Am Diet Assoc 104:1664-1666

3. Singh GK, Kogan MD, van Dyck PC, Siahpush M (2008) Racial/ ethnic, socioeconomic, and behavioral determinants of childhood and adolescent obesity in the United States: analyzing independent and joint associations. Ann Epidemiol 18:682-695

4. Kimbro RT, Brooks-Gunn J, McLanahan S (2007) Racial and ethnic differentials in overweight and obesity among 3-year-old children. Am J Public Health 97:298-305

5. Vohr BR, Boney CM (2008) Gestational diabetes: the forerunner for the development of maternal and childhood obesity and metabolic syndrome? J Matern Fetal Neonatal Med 21:149-157

6. Metzger BE, Lowe LP, Dyer AR et al (2008) Hyperglycemia and adverse pregnancy outcomes. N Engl J Med 358:1991-2002

7. Sacks DA (2007) Etiology, detection, and management of fetal macrosomia in pregnancies complicated by diabetes mellitus. Clin Obstet Gynecol 50:980-989

8. Catalano PM, Hauguel-De MS (2011) Is it time to revisit the Pedersen hypothesis in the face of the obesity epidemic? Am J Obstet Gynecol 204:9

9. Leikin EL, Jenkins JH, Pomerantz GA, Klein L (1987) Abnormal glucose screening tests in pregnancy: a risk factor for fetal macrosomia. Obstet Gynecol 69:570-573

10. Ouzounian JG, Hernandez GD, Korst LM et al (2011) Prepregnancy weight and excess weight gain are risk factors for macrosomia in women with gestational diabetes. J Perinatol 31:717-721

11. Hunt KJ, Marlow NM, Gebregziabher M et al (2012) Impact of maternal diabetes on birthweight is greater in non-Hispanic blacks than in non-Hispanic whites. Diabetologia 55:971-980

12. Zhang J, Troendle J, Reddy UM et al (2010) Contemporary cesarean delivery practice in the United States. Am J Obstet Gynecol 203:326e1-326e 10

13. Institute of Medicine and National Research Council Committee to Reexamine IOM Pregnancy Weight Guidelines, Rasmussen KM, Yaktine AL (eds) (2009) Weight gain during pregnancy: reexamining the guidelines. National Academies Press, Washington, DC, pp 1-324

14. Ehrenberg HM, Mercer BM, Catalano PM (2004) The influence of obesity and diabetes on the prevalence of macrosomia. Am J Obstet Gynecol 191:964-968 
15. Schaefer-Graf UM, Heuer R, Kilavuz O, Pandura A, Henrich W, Vetter K (2002) Maternal obesity not maternal glucose values correlates best with high rates of fetal macrosomia in pregnancies complicated by gestational diabetes. J Perinat Med 30:313-321

16. Bo S, Menato G, Signorile A et al (2003) Obesity or diabetes: what is worse for the mother and for the baby? Diabetes Metabol 29:175-178

17. Black MH, Sacks DA, Xiang AH, Lawrence JM (2013) The relative contribution of prepregnancy overweight and obesity, gestational weight gain, and IADPSG-defined gestational diabetes mellitus to fetal overgrowth. Diabetes Care 36:56-62

18. Dooley SL, Metzger BE, Cho NH (1991) Gestational diabetes mellitus. Influence of race on disease prevalence and perinatal outcome in a U.S. population. Diabetes 40:25-29

19. Berkowitz GS, Lapinski RH, Lee D (1992) Race/ethnicity and other risk factors for gestational diabetes. Am J Epidemiol 135:965-973

20. Kieffer EC, Alexander GR, Kogan MD et al (1998) Influence of diabetes during pregnancy on gestational age-specific newborn weight among US black and US white infants. Am J Epidemiol 147:1053-1061
21. Homko CJ, Sivan E, Nyirjesy P, Reece EA (1995) The interrelationship between ethnicity and gestational diabetes in fetal macrosomia. Diabetes Care 18:1442-1445

22. Silva JK, Kaholokula JK, Ratner R, Mau M (2006) Ethnic differences in perinatal outcome of gestational diabetes mellitus. Diabetes Care 29:2058-2063

23. Cripe SM, O’Brien W, Gelaye B, Williams MA (2012) Perinatal outcomes of Southeast Asians with pregnancies complicated by gestational mellitus or preeclampsia. J Immigr Minor Health $14: 747-753$

24. Shah A, Stotland NE, Cheng YW, Ramos GA, Caughey AB (2011) The association between body mass index and gestational diabetes mellitus varies by race/ethnicity. Am J Perinatol 28:515-520

25. Wang J, Thornton JC, Russell M, Burastero S, Heymsfield S, Pierson RN Jr (1994) Asians have lower body mass index (BMI) but higher percent body fat than do whites: comparisons of anthropometric measurements. Am J Clin Nutr 60:23-28

26. Lovejoy JC, Smith SR, Rood JC (2001) Comparison of regional fat distribution and health risk factors in middle-aged white and African American women: The Healthy Transitions Study. Obes Res 9:10-16 